\title{
REGULARIZATION EFFECTS ON 2-D SEISMIC REFRACTION TOMOGRAPHY: CASE STUDY ON SHALLOW MARINE ENVIRONMENT
}

\author{
Frank Cenci Bulhões ${ }^{1,2}$, Marco Antônio Cetale Santos ${ }^{2}$, Luiz Alberto Santos ${ }^{1,2}$, Victor Thadeu Xavier de Almeida ${ }^{1}$
}

1 Petrobras - Petróleo Brasileiro S.A., 2 Universidade Federal Fluminense

Copyright 2021, SBGf - Sociedade Brasileira de Geofísica

This paper was prepared for presentation during the $17^{\text {th }}$ International Congress of the Brazilian Geophysical Society held in Rio de Janeiro, Brazil, 16-19 August 2021.

Contents of this paper were reviewed by the Technical Committee of the $17^{\text {th }}$ International Congress of the Brazilian Geophysical Society and do not necessarily represent any position of the SBGf, its officers or members. Electronic reproduction or repres of of the Brazilian Geophysical Society is prohibited.

\section{Abstract}

A common approach to stabilize the ill-posed inverse problem is to apply regularization, which corresponds to restrict possible solutions for the inverse problems. Thus, a regularization term is often incorporated into the tomographic error function to resolve the non-uniqueness of the inverse geophysical problem. This work aims to evaluate the refraction tomography algorithm, the regularization effects and analyze their effects on the resulting seismic velocities. The study is based, methodologically, on a case study on the 2-D synthetic seismic survey. The regularizations tested in this work are Tikhonov of order 1 , the variants of order 1 , and order 2 . The tomography program is based on Podvin's ray tracing. Obtaining the travel time matrix uses the finite differences scheme with the eikonal equation for the first arrivals, computation of the tomographic matrix, and the regularization algorithm. The velocity model consists of a synthetic based on a shallow seabed channel geology. The actual model contains three layers and a seabed channel structure, with velocities of $1500 \mathrm{~m} / \mathrm{s}$ (water), $2000 \mathrm{~m} / \mathrm{s}$ and $2500 \mathrm{~m} / \mathrm{s}$ with the results of transit time tomography without regularization with regularization schemes. Transit times for tomography are obtained by forward modeling of this model, with the same tomography algorithm to calculate transit time. We conclude that: 1 ) the results of the tomography show better results with better definition and less distortion of the structures with the application of regularization; 2) Tikhonov regularization of order 2 shows faster convergence with improvement in the velocity model and 3) the parameter sensitivity test shows how much an inappropriate choice can distort geological structures.

\section{Introduction}

Most geophysical studies deal with inverse problems since the acquisition of forward measures of physical properties is costly. Also, in general, forward measures are restricted to areas that are spatially limited and difficult to extrapolate to the entire area of interest with the necessary accuracy, even with the use of geostatistical methods.

In the geophysics of oil and gas exploration, seismic methods have been the most used since the beginning of the 20th century (Telford et al. 1990). At the end of the
20 th century and the beginning of the 21 st, there was a significant advance in the oil and gas industry with the large-scale use of 3-D seismic survey data. The increase in computational capacities has generated great advances, which resulted in a significant impact on deep seismic processing (Woodward et al., 2008). Thus, the seismic velocity models have become fundamental for seismic imaging.

The areas of interest of the oil industry are, in general, at great depths, where the refraction tomography does not reach. However, velocity models in the shallow portions directly impacts imaging and, consequently, on the interpretation of deeper structures. Static correction, which is used in the processing of terrestrial data, depends on the velocity model in the shallow end and is the first source of uncertainties and errors resulting from the seismic processing (Lines and Newrick, 2004).

All available velocity information (tomography, migration, well profiles, etc.) must be used and weighted with geological information, statistical treatments, and geostatistical tools (Maul et al., 2005; Bulhões et al., 2018). In most cases, the well's shallow part is not profiled due to operational (lack of stability) and economic factors (Bulhões et al., 2018). Figure 1 presents the seismic section of cross reflection by three wells and their respective sonic profiles.

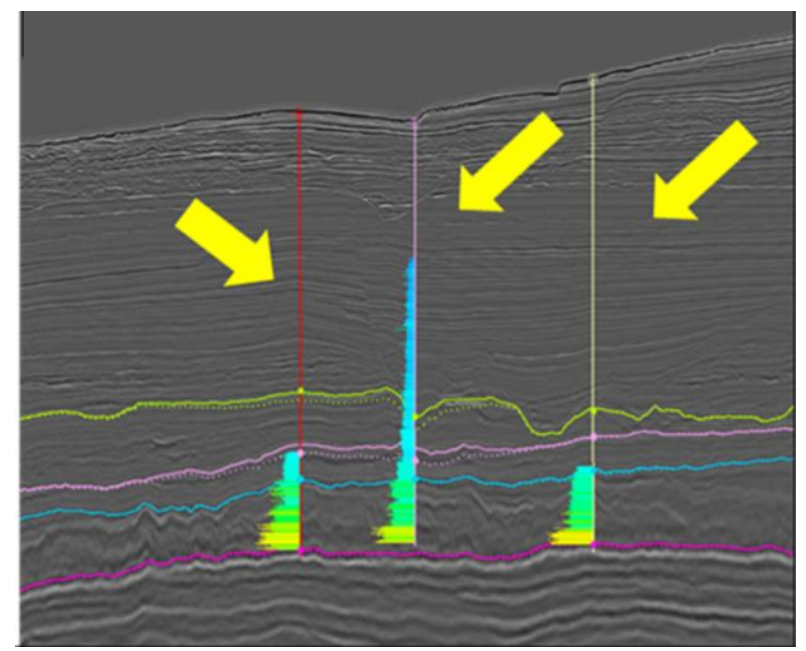

Figure 1: Seismic section that passes through three wells and their respective sonic profiles.

Source: Adapted from Bulhões et al. (2018).

According to Menke (1989), an inverse problem can be defined as a set of mathematical techniques that extract the physical parameters from the corresponding observed data and correlate a physical-mathematical model that justifies them. A problem is said to be the opposite when 
it is desired to infer the medium's physical properties from recorded data that are external to it. A more comprehensive way to define an inverse problem is to consider how to obtain the physical model (set of properties and parameters) from observed data and corresponding observations. This occurs in geophysical and geological interpretation examples.

By the inversion theory, it is defined from Equation 1:

Where:

$$
\boldsymbol{d}=\mathbf{G m} \quad \text { Eq. } 1
$$

$\mathbf{d}$ is the data vector corresponding to an observation; $\mathbf{m}$ is the model parameter;

$\mathbf{G}$ is the matrix that relates the parameters $\mathbf{d}$ and $\mathbf{m}$.

Figure 2 shows schematically what are forward and inverse problems. The inverse problem consists of: having $\mathbf{d}$ and $\boldsymbol{G}$, obtain $\mathrm{m}$. The forward problem is to obtain $\mathbf{d}$ from $\mathrm{m}$ and $\boldsymbol{G}$.

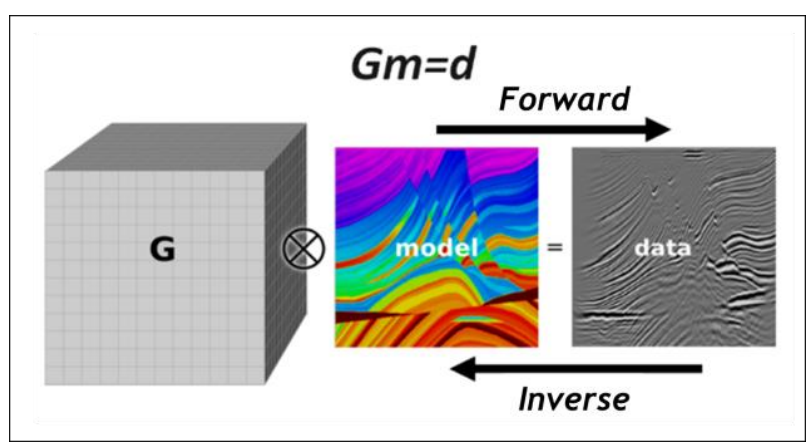

Figure 2: Schematic representation of a forward and inverse problem.

Source: Bianco (2013).

Hadamard (1902) established that an inverse problem is called ill-posed when the solution does not meet at least one of the conditions: existence, uniqueness, and stability. In a well-posed problem, the three conditions mentioned must be satisfied.

According to Zhdanov (2003), all information about the physical model $(\mathrm{m})$ it generates (d), according to Equation 1 , is contained in matrix $G$. However, there are always errors in the measured data, whether in the measurement, noise inherent in the system, or associated with the physical problem. Due to these factors, it is not always possible to find the model, " $m$ ", which describes the registered data, "d", since these errors are not considered in the modeling. According to Aster et al. (2005), there is a possibility that the model adopted to describe the data is not correct (or complete), which also makes it impossible to obtain a solution to the problem.

The least-squares method is an optimization method applied during data inversion. That is, it is a method for solving linear systems, being very efficient in most cases. According to Claerbout and Muir (1973), this type of tool for adjusting curves is relatively sensitive to noise with very pronounced amplitudes. In the case of the non-linear problem, the L2 standard is widely used. Its objective is to minimize a specific function, making the residue, which is the difference between the calculated data and the observed data, be as small as possible and acceptable for a given problem. Thus, in the context of least squares (L2 norm), the objective function to be minimized is that of the square of the residue, given by Equation 2

$$
\varphi(\boldsymbol{m})=\left\|\boldsymbol{d}_{\text {obs }}-\boldsymbol{G}(\boldsymbol{m})\right\|_{2^{2}} \quad \text { Eq. } 2
$$

Where:

$\boldsymbol{d}_{\text {obs }}$ is the observed data; $\boldsymbol{G}(\boldsymbol{m})$ is the calculated data.

Minimizing Equation 2 means looking for the solution that has the smallest possible residue to the observed data and, consequently, obtaining the parameters that best fit the observations.

To minimize the objective function, it must find the points at which your derivative is zero. Thus, by deriving Equation 3 and equaling to zero, one obtains

$$
\boldsymbol{m}=\left[\boldsymbol{G}^{T} \boldsymbol{G}\right]^{-1} \boldsymbol{G}^{T} \boldsymbol{d} \quad \text { Eq. } 3
$$

Substituting $\mathrm{m}$ for $\Delta \mathrm{m}$ gives:

$$
\Delta \boldsymbol{m}=\left[\boldsymbol{G}^{T} \mathrm{G}^{-1} \boldsymbol{G}^{T} \Delta \boldsymbol{d} \text { Eq. } 4\right.
$$

$\Delta \mathrm{m}$ is the velocity model update term. The term depends on the inverse of the $\boldsymbol{G}^{T} \boldsymbol{G}$ matrix. In Equation 5, the updated model $\boldsymbol{m}_{i+1}$

$$
\boldsymbol{m}_{i+1}=\boldsymbol{m}_{\boldsymbol{i}}+\Delta \boldsymbol{m} \text { Eq. } 5
$$

The tomographic process will run a number of predefined iterations is reached or when the residue is less than the stipulated value (Equation 6).

$$
\left\|\boldsymbol{d}-\boldsymbol{G m}_{\boldsymbol{s}}\right\|_{2}{ }^{2}<\delta \quad \text { Eq. } 6
$$

Where:

$\left\|\boldsymbol{d}-\boldsymbol{G m}_{\boldsymbol{s}}\right\|_{2}$ it is the norm of the error between the observed data and the predicted data; and $\delta$ is the value chosen as a tolerance for the error norm between the observed data and the predicted data.

This paper presents a study on the impact of regularization on refraction tomography. The study area represents a coastal environment, close to South Boston, Massachusetts (Zhang and Toksöz 1998), a shallow structure 2-D velocity model. To achieve this objective the paper is divided in the next following topics: In the method topic we describe the experiments; in the results we present, discuss and analyze the employed regularizations; finally we describe the conclusions.

\section{Method}

According to Zhdanov (2003), Tikhonov's work importance is related to the mathematical demonstration that ill-posed problems can be solved. With the continuous $G^{-1}$ operator in this set, it is necessary to know a priori that there is a solution contained in a subset of all possible solutions. When this subset is selected, the illposed problem becomes conditionally well-posed.

In the particular case of some indeterminate linear systems (with several unknowns below the number of equations), a studied solution was to approach the minimum norm's least-squares. That is, there is an 
additional term associated with the quadratic discrepancy term. This can be generalized to solve ill-posed problems, and it is necessary to provide additional information. The regularization method consists of determining the softest approximate solution and checking its compatibility with the observed data for a given noise level.

The purpose of regularization techniques is to make the operator who inverts the data continuously; this is equivalent to making the solutions stable and the problem well-posed. When regularizing the inversion, the problem to be solved is no longer precisely the original problem and becomes a problem that approaches the original (Figure 4). The smoothing factor (or parameter) $(\lambda)$ determines the weight given to smoothing in the inversion process, and according to $\lambda$ tends to 0 the smoothed solution $(\mathbf{m} \lambda)$ must tend asymptotically to the correct solution $m_{c}$ (ZHDANOV, 2003), as well as the smoothed operator must tend to the $G^{-1}$ operator.

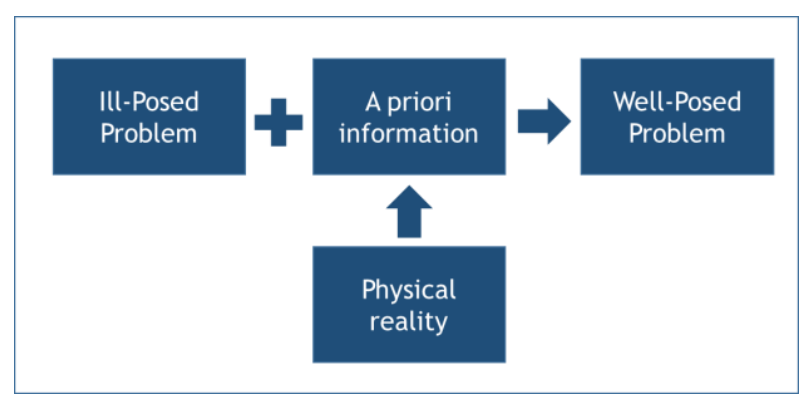

Figure 4: Schematic representation of an ill-posed problem.

Source: Adapted from Velho (2008).

The solution of the inverse problem without regularization is the minimization of $\left\|\boldsymbol{d}-\boldsymbol{G m}_{s}\right\|_{2}$, called objective function (Equation 7):

$$
\min \left\|\boldsymbol{d}-\boldsymbol{G m}_{s}\right\|_{2} \quad \text { Eq. } 7
$$

To obtain a general expression to be minimized, simply apply Lagrange multipliers to the given condition, the result of which is:

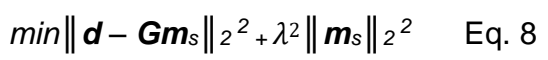

The higher order Tikhonov regularizations follow the same logic as zero order regularization, just replacing the minimization of the $\mathrm{m}_{\mathrm{s}}$ norm by minimizing an $\mathrm{m}_{\mathrm{s}}$ function (Equation 9).

$$
\min \left\|\boldsymbol{d}-\mathbf{G m}_{s}\right\|_{2}{ }^{2}+\lambda^{2}\left\|\boldsymbol{L} \boldsymbol{m}_{s}\right\|_{2}{ }^{2} \quad \text { Eq. } 9
$$

Where:

$\mathbf{L}$ is the matrix of the operator considered (derived first or second from $\mathbf{m}_{\mathbf{s}}$ ).

The Tikhonov regularization of order 1 uses the first derivative of $\mathbf{m}_{\mathbf{s}}$ (Equation 10) and the regularization of order 2, the second derivative (Equation 11). Thus, a more generic equation is:

$$
\begin{gathered}
L^{(1)} m_{s}(x, z)=\left(\frac{\partial m_{s}(x, z)}{\partial x}+\frac{\partial m_{s}(x, z)}{\partial z}\right) \\
L^{(2)} m_{s}(x, z)=\left(\frac{\partial^{2} m_{s}(x, z)}{\partial x^{2}}+\frac{\partial^{2} m_{s}(x, z)}{\partial z^{2}}\right)
\end{gathered}
$$

If the matrix $\mathbf{L}$ is considered as the identity matrix, it is the zero order regularization.

The acquisition geometry for parameterization of the forward modeling is defined as the interval between shooting points in $40 \mathrm{~m}$, the total number of shots 176 , depth of sources and receivers $20 \mathrm{~m}$, the interval between receivers $10 \mathrm{~m}$, the total number of receivers 150 and is represented in Figure 5.

Figure 5 shows the representation with the real velocity model and the acquisition geometry. The actual model contains three layers and a seabed channel structure, with velocities of $1500 \mathrm{~m} / \mathrm{s}$ (water), $2000 \mathrm{~m} / \mathrm{s}$, and 2500 $\mathrm{m} / \mathrm{s}$. In addition, the velocity model consists of $250 \times 25$ cells with a uniform spacing of $10 \mathrm{~m}$. In the study, the actual model was compared with transit time tomography results without regularization with Tikhonov's regularization schemes. Transit times for tomography were obtained by forward modeling of this model, with the same tomography algorithm to calculate transit time.

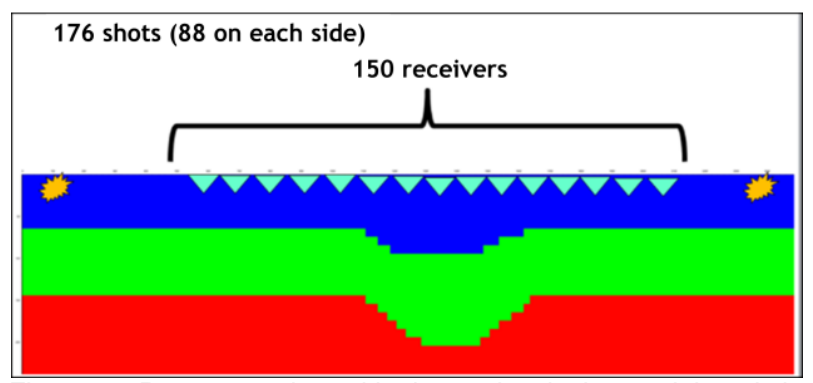

Figure 5: Representation with the real velocity model and the acquisition geometry.

The initial velocity model used for tomographic inversion is shown in Figure 6, consisting of two flat, parallel, and horizontal layers with velocities of $1500 \mathrm{~m} / \mathrm{s}$ and 2500 $\mathrm{m} / \mathrm{s}$.

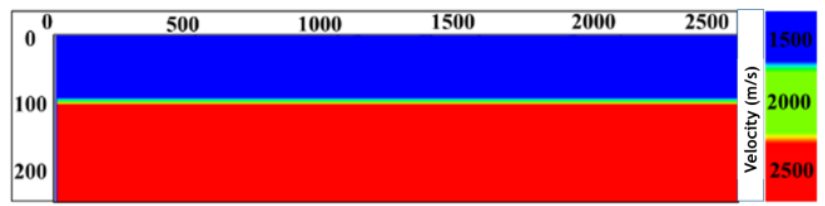

Figure 6: Initial model of entry of refraction tomography.

In Figure 7, the real velocity model is represented with the model resulting from the refraction tomography without applying the regularization. The difference between the real and calculated model is in Figure 7. The residual of the tomographic process as a function of the iteration is shown in Graph 1. The axis referring to the residue is in the logarithmic scale at base 10. It is observed in this graph that the convergence of the tomographic inversion occurs from iteration 28 . 


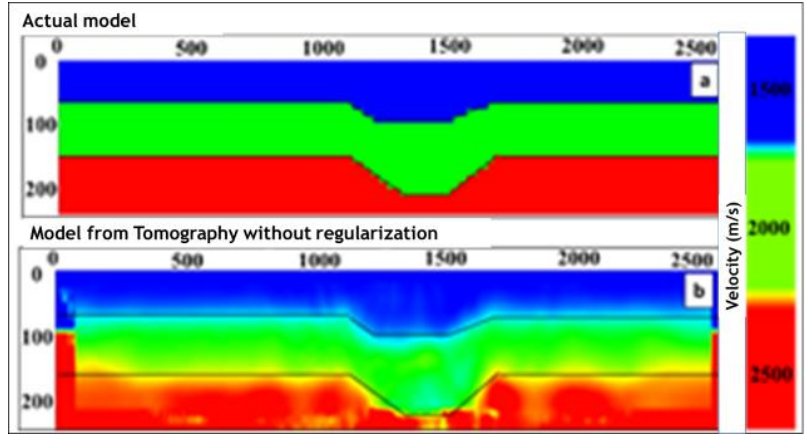

Figure 7: (a) Actual velocity model and (b) Tomography result without applying regularization.

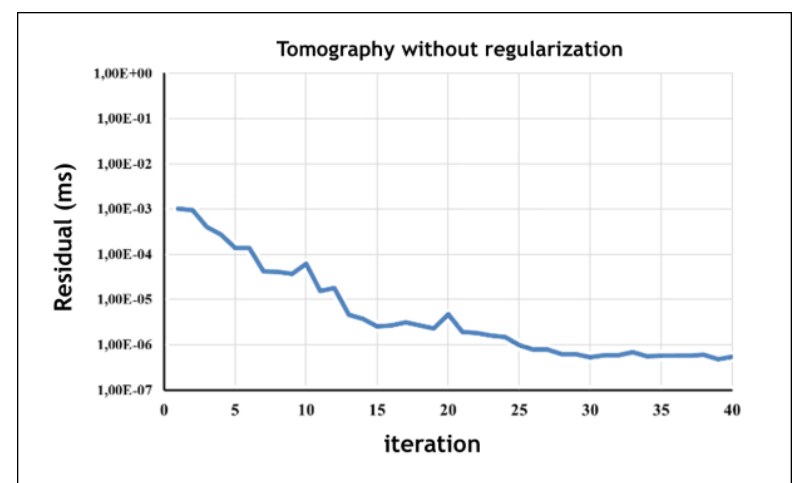

Graph 1: Variation of the residual of the tomographic inversion without regularization according to the iteration.

The analysis of the effects of regularization took place through a sensitivity study based on the regularization parameters, which impact the algorithm's convergence and stability and the resulting model. The stability is analyzed using graphs of the behavior of the residue as a function of iteration.

\section{Examples}

For the analysis of the effect of regularization on refraction tomography in the present work, the program implemented by the Seismic Imaging Group of Universidade Federal Fluminense is used. It is based on Almeida's work (2013), which uses finite differences method to calculate transit time from eikonal equations and complemented by Bulhões (2020).

We apply Tikhonov regularizations of order 1 and order 2 . In the case of order 1, three implementations are applied: (i) conventional (derived in the horizontal and vertical directions), (ii) the derivative in the horizontal component, and (iii) the derivative with the advanced scheme. The tomographic inversion is applied for values of the regularization parameter varying from $10^{-8}$ to $10^{3}$.

In Tikhonov's regularization of order 1 , the term added to stabilize the inverse problem is the first derivative in the $x$ and $z$ directions (and $y$ in the 3-D case) defined by Equation 10.

The second regularization approach is the adaptation of the 1st order Tikhonov regularization, using only the horizontal component of the derived operator. The third approach adopted to implement the Tikhonov regularization of order 1 uses the advanced scheme in the derived operator.

The last approach adopted in the study is the Tikhonov regularization of order 2 . This regularization is the second derivative of the model in the spatial directions (Equation 11).

\section{Results}

Graph 2 shows the residual transit time calculated as a function of the iteration for the regularization parameters. For $\lambda=1000$, numerical instability occurs during the tomographic process. It is also observed that for values less than 1000, the process runs smoothly without sudden variations, and the residue converges to values around $6 \times 10^{-7}$.

To obtain optimal values for regularization is not trivial. There are strategies to be modeled, such as the L-curve, but there is always a need to execute the tomographic inversions and from the results and observe what best fits. In other words, the L-curve has no predictive power. Each physical problem will present an optimal value for the regularization parameter according to the order of magnitude of the modeled property.

Among the regularization cases tested, the optimum values of the parameters $\lambda$ for each scenario are located at the apex of the residue curve as a function of the parameter $\lambda$ and this optimum value for each scenario. Graph 2 shows the RMS residue of the transit times as a function of the parameters.

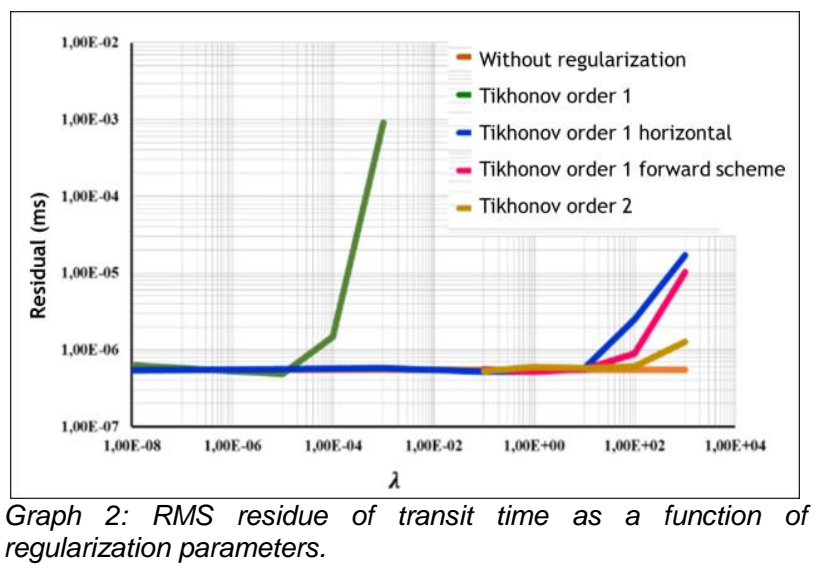

Graph 3 shows the RMS residue variation in transit time as a function of the iteration for the respective optimal values and compared with the tomography without regularization. The convergence of tomography without regularization occurs after 31 iterations. With regularization, convergence occurs in iteration 22 (order 1), 25 (order 1 horizontal component), 28 (order 1 advanced scheme) and 17 (order 2). 


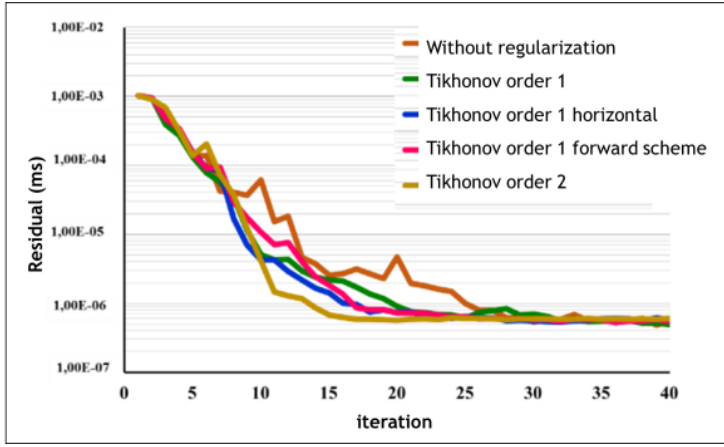

Graph 3: RMS residue of transit time as a function of iteration for cases with and without regularization.

In Figure 8 there are the real models and the tomography results without regularization and the scenarios using the optimal parameterization values. Without regularization, the structures are distorted (Figure 8b). The same distortion occurs in cases of order 1 regularization (Figure $8 \mathrm{c})$. On the other hand, in the cases, order 1 horizontal component (Figure 8d), order 1 with the advanced scheme (Figure 8e), and order 2 (Figure 8f) do not present distortion in the structures.

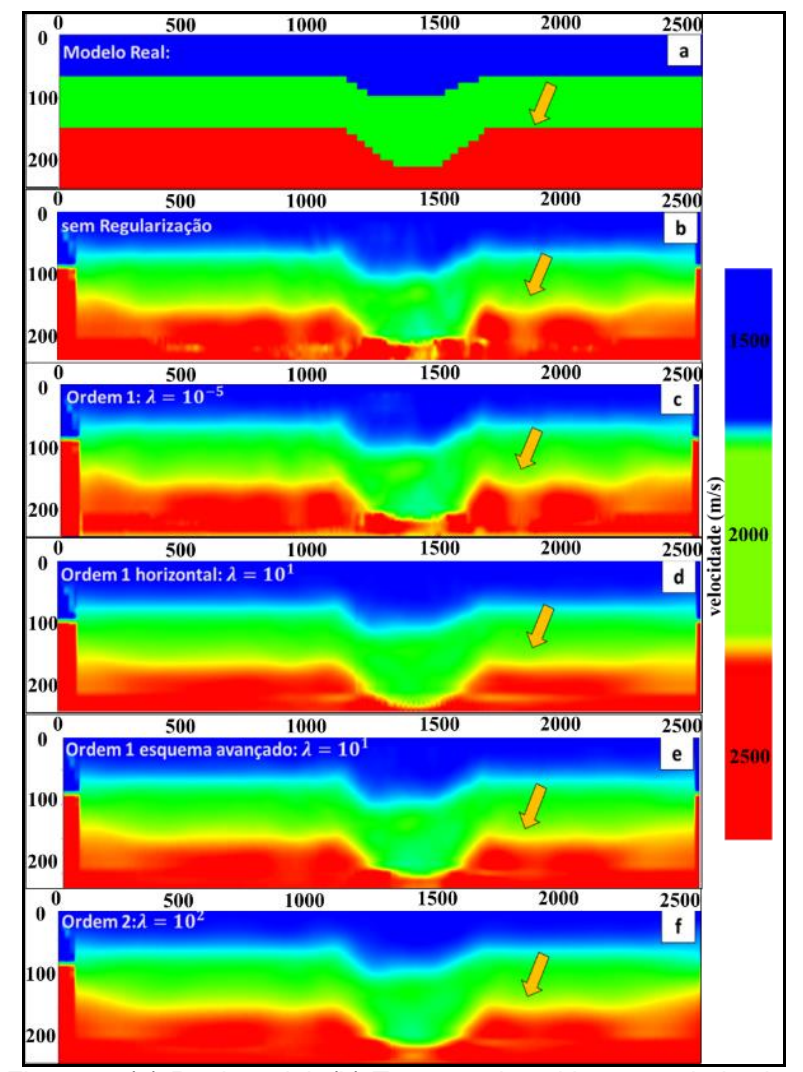

Figure 8: (a) Real model, (b) Tomography without regularization, Tikhonov regularization (c) order 1, (d) order 1 horizontal component, (e) order 1 advanced scheme and $(f)$ order 2.

Table 1 shows the summary of the tested regularizations and the individual results after the 40 iterations of the tomographic process. The Table 1 describes the optimal value of the parameter $\lambda$ for Tikhonov regularization; the step in which the inversion begins to converge to the respective parameter $\lambda$ and the RMS residue of the transit time compared to the actual data, $\delta$.

The use of Tikhonov regularizations of order 2 converges the tomographic process faster from step 17. In the case of the use of Tikhonov regularization of conventional order 1, second line in Table 1, the convergence of the tomography occur in smaller number of steps than without regularization, 31 and 28 respectively.

\begin{tabular}{|l|c|c|c|}
\hline & $\begin{array}{c}\lambda \\
\text { optimum }\end{array}$ & $\begin{array}{c}\text { beginning of } \\
\text { convergence }\end{array}$ & RMS $\delta \mathrm{t}$ \\
\hline Without regularization & - & 28 & $5.47 \mathrm{E}-07$ \\
\hline Tikhonov 1st order & $10^{-5}$ & 31 & $4.88 \mathrm{E}-07$ \\
\hline Tikhonov 1st order horizontal & 10 & 22 & $5.76 \mathrm{E}-07$ \\
\hline $\begin{array}{l}\text { Tikhonov 1st order forward } \\
\text { schema }\end{array}$ & 10 & 25 & $5.54 \mathrm{E}-07$ \\
\hline Tikhonov 2nd order & 100 & 17 & $6.06 \mathrm{E}-07$ \\
\hline
\end{tabular}

Table 1: Summary of the regularizations applied with the respective waste statistics in the 40th iteration.

The best result obtained for this scenario goes against the results obtained in Denisov et al. (2006) and Teimoornega and Poroohan (2010). In both studies, second-order Tikhonov presents better feature delineation than Tikhonov of order 1.

\section{Conclusions}

The study aims to evaluate the algorithm of refraction tomographic inversion in the shallow surface and the effects of regularization on refraction seismic tomography - the effects of this regularization on the resulting seismic velocities.

The analysis carries out that regularization alone is not enough to solve ill-posed problems. The use of regularization or inadequate parameter values does not add a correction to the tomographic result. The sensitivity study results for Tikhonov regularizations shows that there are distortions in the geological structures for a range of values of inadequate regularization parameters.

The velocity model resulting from refraction tomography using classical -order 1 regularization (derived in the $x$ and $z$ directions) does not solve the existing problems without regularization and shows significant numerical instability.

Refraction tomography using order 2 Tikhonov regularization converges faster than the other cases, with 17 iterations. The value of the parameter that results in the optimal model is $\lambda=100$ with fewer structural distortions. Although order 1 with horizontal derivative presents the best result for the models, its convergence is very slow, only after 28 iterations.

\section{Acknowledgments}

The authors would like to thank Petrobras and Universidade Federal Fluminense for allowing the publication of this work. Frank Cenci Bulhões thanks manager Francisco Aquino and Leonardo Matriciano for their support in publishing the work and I thank Bruna Faustino for the support and companionship. 


\section{References}

ALMEIDA, V. T. X. 2013. Tomografia sísmica de superfície: modelagem direta calculando as trajetórias de ondas refratadas. Universidade Federal Fluminense: Dissertação de Mestrado.

ASTER, R. C., BORCHERS, B., THURBER, C. H. 2005. Parameter Estimation and Inverse Problems. [S.I.]: Elsevier Academic Press. 320- p.

BULHÕES, F. C. 2020. Efeitos da Regularização na Tomografia de Refração. Universidade Federal Fluminense: Dissertação de Mestrado.

BULHÕES, F. C., FERREIRA, G. D. and CAPARICA JR., J. F. 2018. Impacts of Seismic Velocity Model Calibration for Time-Depth Conversion: A Case Study. Revista Brasileira de Geofísica 36(4). Sociedade Brasileira de Geofísica. ISSN 0102-261X.

CARBONESI, B. L. 2018. Tomografia de Refração e sua Aplicação na Identificação dos Altos Estruturais do Gráben da Casa de Pedra, RJ. Universidade Federal Fluminense: Projeto Final II em Geofísica apresentado à como exigência para obtenção do título de Bacharel em Geofísica.

CLAERBOUT, J. F., MUIR, F. 1973.Robust modeling with erratic data. Geophysics, Society of Exploration Geophysicists 38(5), p. 826-844.

DENISOV, A. M., KRYLOV, A.S. and SIBANOV, V. N. 2006. Second Order Tikhonov Regularization Method for Image Filtering. Anais do International Conference Graphicon 2006. Novosibirsk Akademgorodok. Russia. Disponível em http://www.graphicon.ru

HADAMARD, J. 1902. Sur les problèmes aux dérivées partielles et leur signification physique. Princeton University Bulletin. [S.I.: s.n.] pp. 49-52

LINES, L. e NEWRICK, R. 2004. Fundamentals of Geophysical Interpretation. Society of Exploration Geophysicists. Primeira edição. 288 p. ISSN 978-156080-125-2. Disponível em: <http://dx.doi.org/10.1190/1.9781560801726>.

MENKE, W. 1989. Geophysical Data Analysis: Discrete Inverse Theory. Edição revista. [S.I.]: Academic Press Elsevier. 289 p.

PODVIN, P. and LECOMTE, I. 1991. Finite difference computation of travel times in very contrasted velocity models: a massively parallel approach and its associated tools. Geophysical Journal International. 105, p. 271-284.

MAUL, A., FEILHABER, M. and GONÇALVES, P.A. 2005. Modelagem Geológica de Velocidades Sísmicas Utilizando o GoCad. Material Interno Petrobras.

SHERIFF, R. E. 1995. Exploration Seismology. Segunda edição. Nova York, EUA: Cambridge University Press. $628 \mathrm{p}$.

TEIMOORNEGA, K., POROOHAN, N. 2010. The Application of Discrete Tikhonov Regularization Inverse Problem in Seismic Tomography. Water \& Geoscience. Anais do 4th IASME/WSEAS International Conference on
GEOLOGY and SEISMOLOGY. University of Cambridge, UK February 23-25, 2010.

TELFORD, W. M., GELDART, L. P. and SHERIFF, R. E. 1995. Applied Geophysics. Segunda edição. Nova York, EUA: Cambridge University Press. 1990. 792p.

VELHO, H. F. C. 2008. Introdução aos Problemas Inversos: Aplicações em Pesquisa Espacial. Escola de verão em Computação Aplicada - LAC-INPE 2008. Laboratório Associado de Computação e Matemática Aplicada Instituto Nacional de Pesquisas Espaciais.

WOODWARD, M., NICHOLS, D., ZDRAVEVA, O., WHITFIELD, P. and JOHNS, T. 2008. A decade of tomography. Geophysics. Society of Exploration Geophysicists 73(5). ISSN 0016-8033. Disponível em: <http://dx.doi.org/10- .1190/1.2969907>.

ZHANG, J. and TOKSöZ, M. 1998. Nonlinear refraction travel time tomography. Geophysics. Society of Exploration Geophysicists, 63(5), p. 1726-1737, set. 1998. ISSN 0016-8033. Disponível em: $<$ http://dx.doi.org/10.1190/1.1444468>.

ZHDANOV, M. S. 2003. Tutorial: Regularization in inversion theory: CWP Report: Colorado School of Mines, $136,47$. 